\title{
MID-INFRARED SPECTROSCOPY FOR THE ANALYSIS OF POTASSIUM SOIL PROPERTIES
}

\author{
1D.A. Jakkan, ${ }^{2}$ Chandrasekhar Sakode, ${ }^{3}$ Pradnya H. Ghare \\ 1. Research Scholar, ECE Department, Indian Institute of Information Technology, \\ Nagpur. Assistant Professor, ECE Department, Indian Institute of Information \\ Technology, Nagpur, \\ 2. Assistant Professor, ECE Department, VNIT, Nagpur, \\ dtei18ece001@iiitn.acin, phghare@ece.vnit.ac.in, chandrashekhar.sakode@iiitn.ac.in
}

\begin{abstract}
:
The agricultural sector plays a major role in a country within the economy of the nation. In recent years, many innovations have also been introduced in the field of the agricultural industry. Serious research is happening all over the world, with the invention of IoT, Big Data Technology, and Machine learning how the farmers and even the Govt. Entities will help improve this sector with rising demand for agricultural products worldwide. Various soil properties like ph level, total nitrogen, phosphorus, potassium, carbon contents in soil impacts on crop production in the agriculture sector. Methods for rapid and accurate soil tests are needed for detecting levels of above index properties of soil attributes which help the farmer to take correct decision for crop production. Mid- Infrared Spectroscopy (MIR) is one such technique used to identify the classes and properties of soils with high precision. In this research work, we are using machine learning model for analysis and prediction of Potassium property by using Mid-infrared spectroscopy dataset collected from a various sample of California state in the United States. Our results show that the decision tree regression and random forest regression model gives better accuracy and through that, we can predict the Potassium level of the soil for helping the farmer for getting right kind of crop in that soil sample.
\end{abstract}

Key words: - Soil testing, MIR Spectroscopy, Machine Learning, phosphorus properties.

\section{INTRODUCTION:}

Soil analysis and testing using conventional laboratory technology may be prohibitively achieved (for example, wet chemistry) are costly and often time-consuming, which encourages to transfer functions as a substitution [1][2]. As a result, these costly soil analyses are mostly reduced to a few samples or samples bulked around a region to provide representative composites. These data provide little to no spatial variability knowledge on the soil. To satisfy this need, fast and cost-effective methods must be created and alternative methods based on UV, visible (Vis), nearinfrared (NIR), and mid-infrared (MIR) frequency area (or combinations) data have strong potential. [3][4].
Diffuse Reflection Spectroscopy is a process that can be used to endorse or substitute traditional practices Analysis of the ground. This process has lost a lot overcoming growth over the past two decades' certain limits and a broader range of versatile Statistical models that can be more reliable Align spectrum variations with the variability of Soil's features. Fast and convenient soil spectroscopy is less expensive, non-destructive, simple and, at times, more accurate, Intensified with the use of others rather than conventional analysis Techniques such as geo statistics and multivariate statistics[5]. The strategies involved in the use It is possible to adjust soil spectroscopy for use by researchers, Technicians and farmers, thus providing an adequate supply of Alternative for the 
investigation of soil spatial variability properties, as well as the features and growth of farm crops.

Infrared (IR) spectroscopy can, at present, be summarized as spectra, which are interlinked by means afterwards, Models of calibration based on the behaviour of the Interest properties in samples and with the data obtained and a mathematical model is created that is used for

The prediction of the characteristics of the specimens which were not used for model calibration purposes[6].

This technique is a non-destructive, fast one. One spectrum only Enables the simultaneous establishment of several physical properties of crops and biochemical properties of Soils[7]. It is also less costly. As opposed to conventional laboratory analyses, particularly when it is a large number of samples need to be analysed about the IR [8][9]. The additional benefit of the technique is the ability to use Spectral data as an indicator of the integration of The quality of the soil and, therefore, can be used as a tool for the evaluation of the soil conditions.

Mid-infrared (MIR) spectroscopy is one of the most important analytical tools used to obtain real-time information on the qualitative and quantitative dimensions of analytic[10]. The wavelength range is 400 to $4,000 \mathrm{~cm}-1$, which is equal to a wavelength of 2.5 to $25 \mathrm{~nm}$. This selection is highly important because it offers direct knowledge of the components of a sample and its distinctive molecular structure [11]. While NIR is used more widely, due to the specificity of the absorption bands in this spectral range, MIR spectroscopy is becoming more general [12].Linker demonstrated that mid-infrared spectroscopy can be used to distinguish agricultural soil types consistent with soil characteristics (for example, calcium carbonate minerals, clay, and organic components)[13]. This identification of soil types aims to dramatically improve the determination of nitrate bases in the soil[13].Different experiments have shown how data models of MIR sensors outperform those that use vis-NIR. This can be explained by the fact that the basic molecular vibrations of soil components exist in the MIR range, while the VIS-NIR range senses only their overtones and variations [8]. Consequently, the MIR multivariate calibrations are more stable, which makes sense since the key constituents of soils are organic carbon and clay minerals and have well-defined absorption regions, especially in the MIR range. The present research aimed to evaluate the potential of mid-infrared reflectance (MIR) spectroscopy for the analysis and prediction of Potassium in various agriculture soil samples in California state of United State of America (USA).

\section{MATERIAL \& METHODS:}

\subsection{Database for model development}

For this research study, we had obtained the data from The USDA NSSC-KSSL soil spectral library and associated SCD (as of June 2018). This actual data contains more than 50,000 spectral data of various soils across the different states of the United State of America (USA). Diffuse MIR reflectance spectrometers were acquired using a Bruker Vertex 70 FTIR spectrometer with a high-performance HTS-XS Diffuse Reflectance accessory for air-dried, seven $(2 \mathrm{~mm})$ and ball-milled soil samples[14]. Each sample of soil was quadruplicate screened and the spectra were combined to provide a representative spectrum for each sample of soil. For the experimental purpose, we had taken data for analysing Potassium property from the soil sample of California state. Total of 416 soil samples and their 
measured value of potassium is available in this dataset.

\subsection{Model Selection}

Several machine learning algorithms under the supervised category are available for analysing and checking the model performance on this numerical dataset available for potassium. We had considered only the Soil Id i.e. soil sample and its measured potassium value from the available dataset for simplicity. The train test split is kept as a $70-30 \%$ ratio, so that model will learn from more data. We had applied all possible regression model of machine learning which includes decision tree, random forest[15], linear regression[15], support vector machine regression and compared this model on different parameter Root mean square error (RMSE)and accuracy score for training and testing dataset.

After analysing above soil spectra, as the spectroscopy range increases the observed values of potassium property is getting diminished. The potassium property values are better observed when the value of reflectance is between $1750-750 \mathrm{~nm}$. The best or pick value is observed at an approx. value of 847 $\mathrm{nm}$ reflectance of spectroscopy.

\section{RESULT \& DISCUSSION:}

\subsection{Model Performance}

After applying several machine learning models on the available dataset, the comparative analysis of performance is shown in below table 1 .

From table 2 it is analysed after performing several machine learning algorithms on an available dataset of potassium soil property that Decision Tree and Random Forest algorithm perform better compared to another algorithm. The RMSE values for decision tree for training and the testing dataset are 0.00 and 4.70 respectively whereas for random forest algorithm it is 2.76 and 4.43 for training and testing dataset respectively. Hence by observing all values we can conclude that the random forest algorithm performs better for analysing and predicting potassium soil property in the state of California.

\section{CONCLUSION:}

With the help of MIR Infrared spectroscopy, various soil properties were demonstrated in related spectral regions, but with the dissimilar level of spectroscopy reflectance range.

Through this research and results drawn after applying Machine Learning models, by using mid-infrared (MIR) reflectance spectroscopy, a large number of soil samples to identify various properties like potassium, carbon, nitrogen can be identified. Although we had analysed potassium property in this work and found that random forest gives better performance on this soil sample from California state, but in future, we would like to perform various calibration and testing of different properties on multiple soil samples from the different state of USA. We would also like to focus on MIR Spectroscopy range for different soil properties as future work.

\section{REFERENCES:}

K. E. Saxton, W. J. Rawls, J. S. Romberger, and R. I. Papendick, "Estimating Generalized Soil-water Characteristics from Texture," Soil Sci. Soc. Am. J., vol. 50, no. 4, pp. 1031-1036, 1986, doi: $10.2136 /$ sssaj 1986.036159950050000400 39x.

B. Minasny, A. B. McBratney, and K. L. Bristow, "Comparison of different approaches to the development of pedotransfer functions for water-retention curves," Geoderma, vol. 93, 
no. 3-4, pp. 225-253, 1999, doi: 10.1016/s0016-7061(99)00061-0.

L. J. Janik, R. H. Merry, and J. O. Skjemstad, "Can mid infrared diffuse reflectance analysis replace soil extractions?," Aust. J. Exp. Agric., vol. 38, no. 7, p. 681, 1998, doi: 10.1071/ea97144.

R. A. Viscarra Rossel, V. I. Adamchuk, K. A. Sudduth, N. J. McKenzie, and C. Lobsey, "Proximal Soil Sensing: An Effective Approach for Soil Measurements in Space and Time," Advances in Agronomy. Elsevier, pp. 243-291, 2011, doi: 10.1016/b978-012-386473-4.00005-1.

P. TITTONELL, K. SHEPHERD, B. VANLAUWE, and K. GILLER, "Unravelling the effects of soil and crop management on maize productivity in smallholder agricultural systems of western Kenya-An application of classification and regression tree analysis," Agric. Ecosyst. Environ., vol. 123, no. 1-3, pp. 137-150, 2008, doi: 10.1016/j.agee.2007.05.005.

J. G. Cobo et al., "Integration of mid-infrared spectroscopy and geostatistics in the assessment of soil spatial variability at landscape level," Geoderma, vol. 158, no. 34, pp. 398-411, 2010, doi: 10.1016/j.geoderma.2010.06.013.

D. Cozzolino and A. Morón, "Potential of nearinfrared reflectance spectroscopy and chemometrics to predict soil organic carbon fractions," Soil Tillage Res., vol. 85, no. 1-2, pp. 2006, $78-85, \quad$ doi: 10.1016/j.still.2004.12.006.

R. A. Viscarra Rossel, D. J. J. Walvoort, A. B. McBratney, L. J. Janik, and J. O. Skjemstad, "Visible, near infrared, mid infrared or combined diffuse reflectance spectroscopy for simultaneous assessment of various soil properties," Geoderma, vol.
131, no. 1-2, pp. 59-75, 2006, doi: 10.1016/j.geoderma.2005.03.007.

J. H. Camacho-Tamayo, Y. Rubiano S., and M. del P. Hurtado S., "Near-infrared (NIR) diffuse reflectance spectroscopy for the prediction of carbon and nitrogen in an Oxisol," Agron. Colomb., vol. 32, no. 1, pp. 86-94, 2014, doi: 10.15446/agron.colomb.v32n1.38967.

J. P. Bonett, J. H. Camacho-Tamayo, and L. Ramírez-López, "Mid-infrared spectroscopy for the estimation of some soil properties Espectroscopia de infrarrojo medio para la estimación de algunas propiedades del suelo," Agron. Colomb., vol. 33, no. 1, pp. 99-106, 2015, doi: 10.15446/agron.colomb.v33n 1.49245.

Y. Etzion, R. Linker, U. Cogan, and I. Shmulevich, "Determination of Protein Concentration in Raw Milk by Mid-Infrared Fourier Transform Infrared/Attenuated Total Reflectance Spectroscopy," J. Dairy Sci., vol. 87, no. 9, pp. 2779-2788, 2004, doi: 10.3168/jds.s0022-0302(04)73405-0.

C. Du, R. Linker, and A. Shaviv, "Identification of agricultural Mediterranean soils using mid-infrared photoacoustic spectroscopy," Geoderma, vol. 143, no. 1-2, pp. 85-90, 2008, doi: 10.1016/j.geoderma.2007.10.012.

R. Linker, M. Weiner, I. Shmulevich, and A. Shaviv, "Nitrate Determination in Soil Pastes using Attenuated Total Reflectance Mid-infrared Spectroscopy: Improved Accuracy via Soil Identification," Biosyst. Eng., vol. 94, no. 1, pp. 111-118, 2006, doi: 10.1016/j.biosystemseng.2006.01.014.

J. Sanderman, K. Savage, and S. R. S. Dangal, "Mid-infrared spectroscopy for prediction of soil health indicators in the United States," Soil Sci. Soc. Am. J., vol. 84, no. 1, pp. 251261, 2020, doi: 10.1002/saj2.20009. 
M. Mohammed, M. B. Khan, and E. B. M.

Bashie, Machine learning: Algorithms and

applications, no. December. 2016.

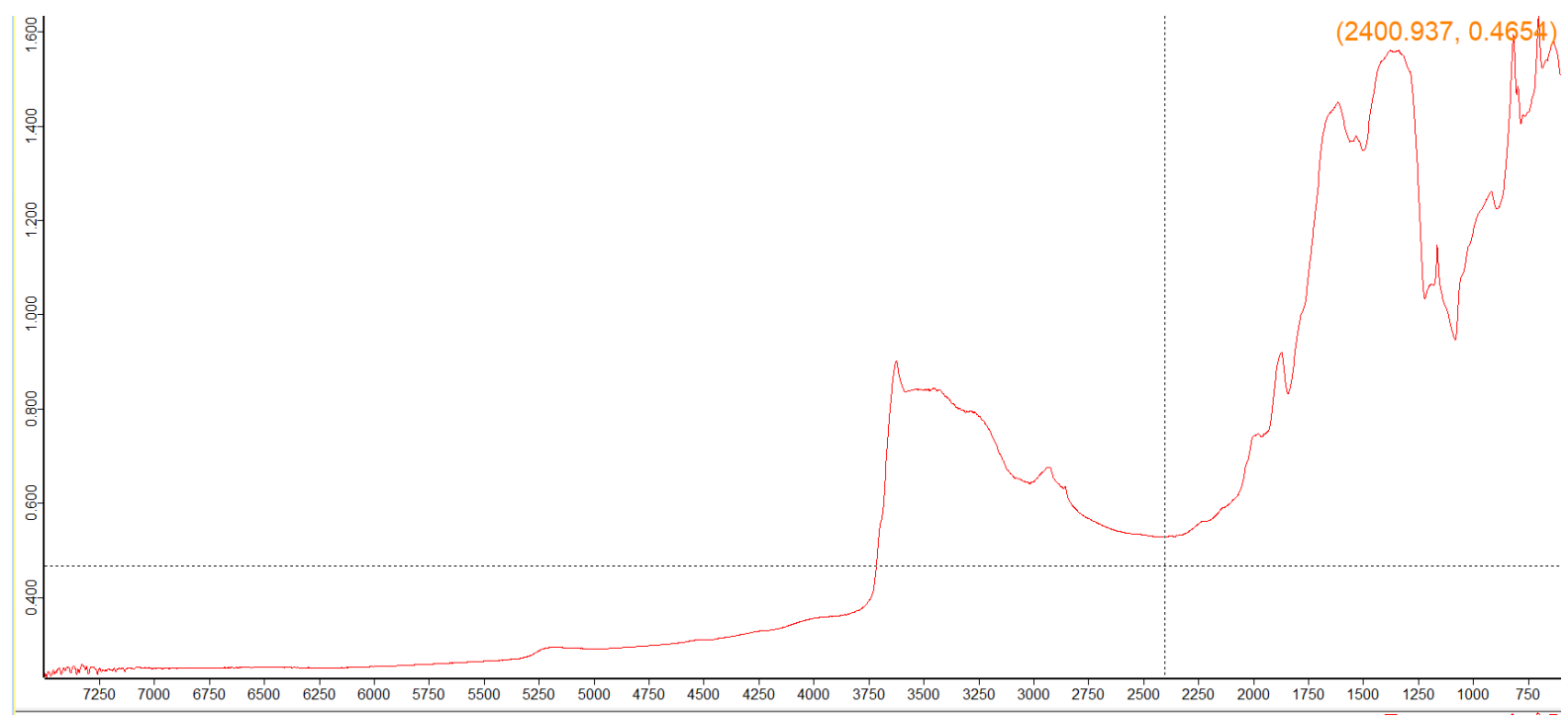

Figure 1: Soil Spectra

Table 1: Calibration and validation results on potassium soil properties

\begin{tabular}{|l|c|c|c|c|}
\hline Model & Accuracy(train) & Accuracy(test) & RMSE(train) & RMSE(test) \\
\hline Decision Tree & 100.0 & 85.15 & 0.00 & 4.70 \\
\hline Random Forest & 98.56 & 88.23 & 2.76 & 4.43 \\
\hline Linear Regression & 19.60 & 18.69 & 7.94 & 7.61 \\
\hline Support Vector & 1.525 & 8.61 & 8.01 & 7.73 \\
\hline
\end{tabular}

